\title{
ELECTRONIC STRUCTURE AND MAGNETISM OF Fe-BASED SUPERCONDUCTORS
}

\author{
G.E. GRECHNEV ${ }^{1}$ A.S. PANFILOV, ${ }^{1}$ A.V. FEDORCHENKO, ${ }^{1}$ \\ V.A. DESNENKO, ${ }^{1}$ I.P. ZHURAVLEVA, ${ }^{1}$ S.L. GNATCHENKO, ${ }^{1}$ \\ D.A. CHAREEV ${ }^{2}$ O.S. VOLKOVA, ${ }^{3}$ A.N. VASILIEV ${ }^{3}$
}

\author{
${ }^{1}$ B. Verkin Institute for Low Temperature Physics and Engineering, \\ Nat. Acad. of Sci. of Ukraine \\ (4\%, Lenin Ave., Kharkov 61103, Ukraine) \\ ${ }^{2}$ Institute of Experimental Mineralogy, Russian Academy of Sciences \\ (Chernogolovka 142432, Moscow District, Russia) \\ ${ }^{3}$ Moscow State University, Physics Department \\ (Moscow 119991, Russia)
}

PACS 74.70.Xa, 74.62.Fj, 75.10.Lp, 75.30.Cr (C) 2012

\begin{abstract}
$A b$ initio calculations of the electronic structures are carried out for the novel $\mathrm{FeSe}_{1-x} \mathrm{Te}_{x}$ superconductors to explain the experimentally found anomalous magnetic properties in the normal state. The calculations have shown that $\mathrm{FeSe}_{1-x} \mathrm{Te}_{x}$ systems are close to a magnetic instability with dominating enhanced spin paramagnetism. The magnetic susceptibility is found to increase gradually with the Te content. The temperature dependences of the magnetic susceptibility $\chi$ and its anisotropy $\Delta \chi=\chi_{\|}-\chi_{\perp}$ are investigated for FeSe, and a growth of the susceptibility with the temperature is revealed in the temperature range $4.2-300 \mathrm{~K}$. For FeTe, a substantial increase of $\chi$ under pressure is found. The calculated paramagnetic susceptibility exhibits a strong dependence on the unit cell volume $V$ and especially the height of chalcogen species from the Fe plane. The calculations have explained the experimental data on $\chi(T)$ and $\chi(P)$ for FeSe and FeTe, respectively.
\end{abstract}

\section{Introduction}

For the last years, the superconducting $\mathrm{FeSe}_{1-x} \mathrm{Te}_{x}$ compounds have attracted the extensive attention [1-4] due to the simplest crystal structure among the new families of iron-based layered compounds exhibiting superconductivity. The structural simplicity favors theoretical studies of chemical substitution and high-pressure effects, which are aimed at promoting a better understanding of a mechanism of the superconductivity.

For the $\mathrm{FeSe}_{1-x} \mathrm{Te}_{x}$ family, a noticeable increase of the superconducting transition temperature with $x$ was found, from $T_{\mathrm{C}} \sim 8 \mathrm{~K}$ at $x=0$ to a value of $\sim 15 \mathrm{~K}$ at $x \simeq 0.5$. Additionally, a large enhancement of $T_{\mathrm{C}}$ up to $35-37 \mathrm{~K}$ was observed in FeSe under high pressures $[5,6]$. Similar pressure effects on $T_{\mathrm{C}}$ were also reported for $\mathrm{FeSe}_{0.5} \mathrm{Te}_{0.5}[7]$.
The FeTe compound is not superconducting, but it exhibits unusual magnetic properties. A sharp drop in the temperature dependence of its magnetic susceptibility $\chi(T)$ with decreasing temperature was detected at $T \simeq 70 \mathrm{~K}$, which is related to a first-order structural phase transition accompanied by the onset of the antiferromagnetic (AFM) order [3,8]. At low temperatures, a monoclinic structure and a commensurate AFM ordering were detected. The origin of the strong correlation between the structural and magnetic transitions in $\mathrm{Fe}_{1+x} \mathrm{Te}$ is not yet clear.

There is a considerable controversy regarding the interplay between electronic structure, magnetism, and superconductivity in $\mathrm{FeSe}_{1-x} \mathrm{Te}_{x}$ compounds, and their magnetic properties are neither well characterized, nor understood. The experimental data on the magnetic susceptibility behavior of $\mathrm{FeSe}_{1-x} \mathrm{Te}_{x}$ systems in the normal state are incomplete and contradicting $[3,8,9]$. In addition, the magnetic behaviors of $\mathrm{FeSe}_{1-x} \mathrm{Te}_{x}$ samples are related to the presence of magnetic impurities and secondary phases. In order to elucidate the role of expected spin fluctuations in superconductivity, it is very important to obtain the intrinsic susceptibility of the Febased superconductors and investigate its evolution with doping, temperature, and pressure.

Here, we report the experimental results on magnetic properties of a series of $\mathrm{FeSe}_{1-x} \mathrm{Te}_{x}$ compounds in the normal state. The experimental studies are supplemented by $a b$ initio calculations of the electronic structure and the magnetic susceptibility of FeSe and FeTe within the density functional theory (DFT). The aim of this study was to shed more light on the relation between magnetic properties and the chemical and structural compositions, as well as the interplay between the 


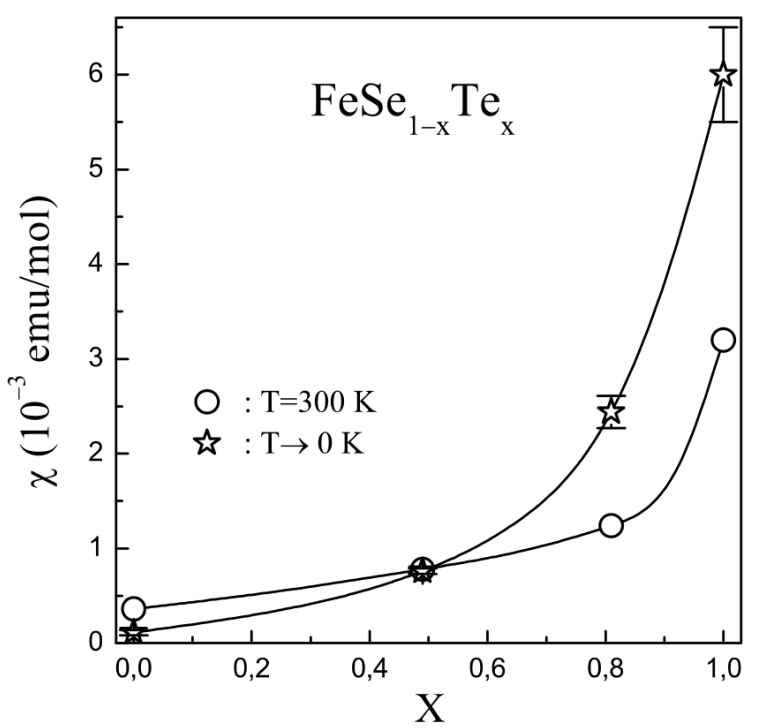

Fig. 1. Concentration dependence of the magnetic susceptibility in the normal state for $\mathrm{FeSe}_{1-x} \mathrm{Te}_{x}$ alloys at $T=300 \mathrm{~K}$ and $T \rightarrow 0 \mathrm{~K}$. Data for FeTe at $T \rightarrow 0 \mathrm{~K}$ are estimated by the extrapolation to zero temperature of the $\chi(T)$ dependence for the paramagnetic state. Solid lines are guides for the eye

onset of superconductivity and magnetic instabilities in the $\mathrm{FeSe}_{1-x} \mathrm{Te}_{x}$ system.

\section{Experimental Details and Results}

Polycrystalline $\mathrm{FeSe}_{1-x} \mathrm{Te}_{x}$ samples were prepared by the conventional solid-state synthesis. The starting chemicals were powder iron (Merck, 99.5\%, $10 \mathrm{~lm}$ ) and crystalline selenium and tellurium cleaned by the floating zone method. These chemicals were mixed in proportions consistent with the reaction stoichiometry, sealed in an evacuated $\left(10^{-4}\right.$ bar) silica glass capsule, and annealed at $700 \mathrm{~K}$ for 14 days. The reacted mixture was ground in an agate mortar under acetone and then pressed into pellets $6 \mathrm{~mm}$ in diameter at a load of 1-1.2 tons, followed by the annealing in an evacuated silica glass capsule at $700 \mathrm{~K}$ for 20 days. The single crystals were grown by the slow-cooling self-flux method. One of the FeSe single crystalline samples was stabilized with a small amount of Co (about $1 \%$ ). The phase content of the samples was checked by the x-ray diffraction technique.

To examine the samples for the presence of ferromagnetic (FM) impurities, the measurements of the DC magnetization $M$ were carried out at $T=5 \mathrm{~K}$ with a magnetic field $H$ up to $50 \mathrm{kOe}$ using a SQUID magnetometer. The experimental superconducting characteristics obtained for the $\mathrm{FeSe}_{1-x} \mathrm{Te}_{x}$ compounds agree

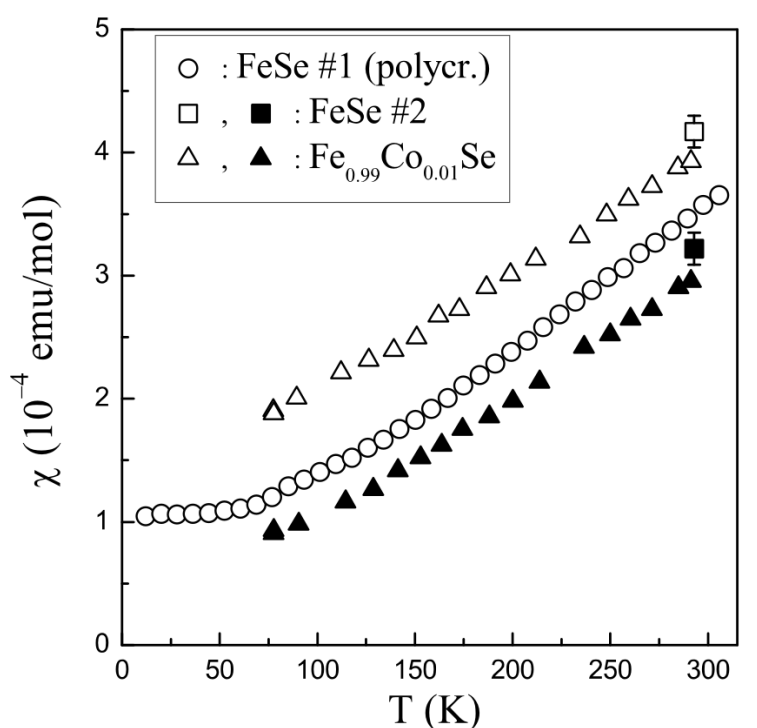

Fig. 2. Temperature dependences of the magnetic susceptibility in the normal state for the polycrystalline sample of FeSe $(\# 1)$ and single-crystalline samples FeSe $(\# 2)$ and $\mathrm{Fe}_{0.99} \mathrm{Co} .01$ Se. Data for single crystals corresponding to $H \| a b$ plane and $H \| c$ are denoted by the open and full symbols, respectively

with those reported in [8-10]. The $M(H)$ dependences for the majority of samples appeared to be quite linear, indicating that the concentration of FM impurities in these samples was negligibly small. For few $\mathrm{FeSe}_{1-x} \mathrm{Te}_{x}$ samples, the magnetization data were corrected for ferromagnetic impurities by subtracting the saturation moment $m_{s}$ from the measured magnetic moment $M(T)$. The former is assumed to be weakly dependent on $T$ up to room temperature [4]. As is seen in Fig. 1, the intrinsic magnetic susceptibility in the series of iron chalcogenides $\mathrm{FeSe}_{1-x} \mathrm{Te}_{x}$ was estimated to increase gradually with the Te content by about ten times.

The measurements of the temperature dependences $\chi(T)$ for FeSe and the susceptibility anisotropy of single crystalline samples were carried out by the Faraday method in the temperature range $78-300 \mathrm{~K}$ and in the magnetic field $H \simeq 1 \mathrm{~T}$. The results of these measurements are presented in Fig. 2. As seen in Fig. 3, the $\chi(T)$ dependence for FeTe exhibits an anomaly at $T \simeq 70 \mathrm{~K}$, which is apparently related to the magnetic and structural transitions [3,8-10]. In the paramagnetic state, the $\chi(T)$ behavior is close to the Curie-Weiss law, and the values $\Theta \simeq-240 \mathrm{~K}$ and $\mu_{\mathrm{eff}} \simeq 3.70 \mu_{B} /$ Fe were estimated.

The study of the magnetic susceptibility under the helium gas pressure $P$ up to 2 kbar was performed at temperatures 55,78 , and $300 \mathrm{~K}$ using a pendulum-type magnetometer placed directly in the nonmagnetic pres- 


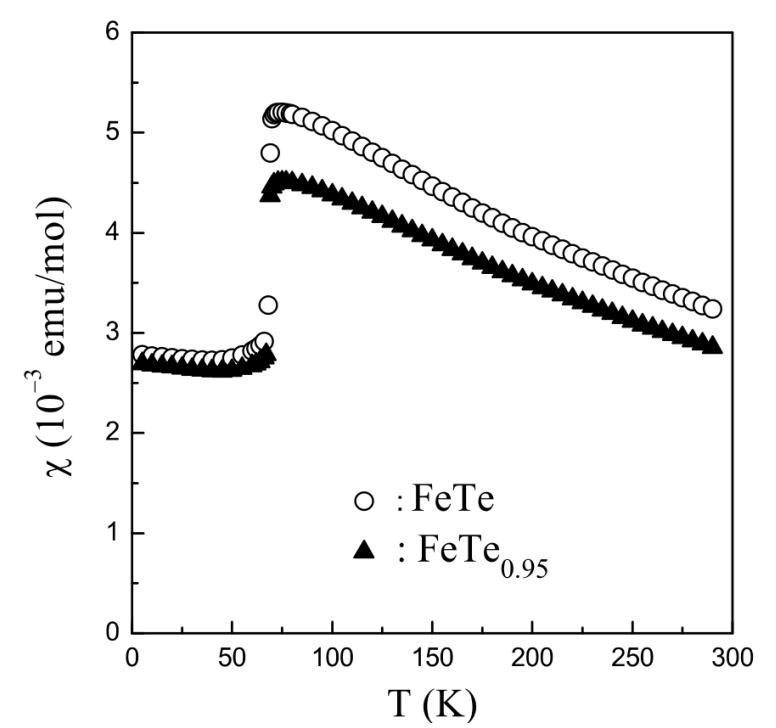

Fig. 3. Temperature dependences of the magnetic susceptibility for single-crystalline samples of FeTe

sure cell [11]. The measurements were carried out in the field $H=1.7 \mathrm{~T}$, and their relative errors did not exceed $0.05 \%$. The large positive values of the pressure effect, $\mathrm{dln} \chi / \mathrm{d} P \simeq 15 \div 30 \mathrm{Mbar}^{-1}$, were observed in the temperature range $78-300 \mathrm{~K}$.

\section{Computational Details and Results}

To understand the magnetic properties of FeSe(Te) compounds in the normal state, the first-principles calculations of the electronic structure and the paramagnetic susceptibility were performed for FeSe and FeTe. Under ambient conditions, $\mathrm{FeSe}(\mathrm{Te})$ compounds possess the tetragonal PbO-type crystal structure, which is composed by alternating triple-layer slabs. Each iron layer is sandwiched between two nearest-neighbor chalcogen layers, which form edge-shared tetrahedra around the iron sites. The positions of Se (or Te) sheets are fixed by the internal parameter $Z$, which represents the height of chalcogen atoms above the iron plane. The structural parameters of $\mathrm{FeSe}(\mathrm{Te})$ compounds were established by means of x-ray and neutron diffraction [2, 3, 8, 9, 12].

The previous calculations of the electronic structure of iron-based superconductors were predominantly related to studies of the AFM and SDW ordering [13-15]. The aim of this work was to investigate the paramagnetic response in an external magnetic field and to elucidate the nature of paramagnetism in the parent FeSe and FeTe compounds. The dependences of the magnetic susceptibility on the volume, lattice parameters,

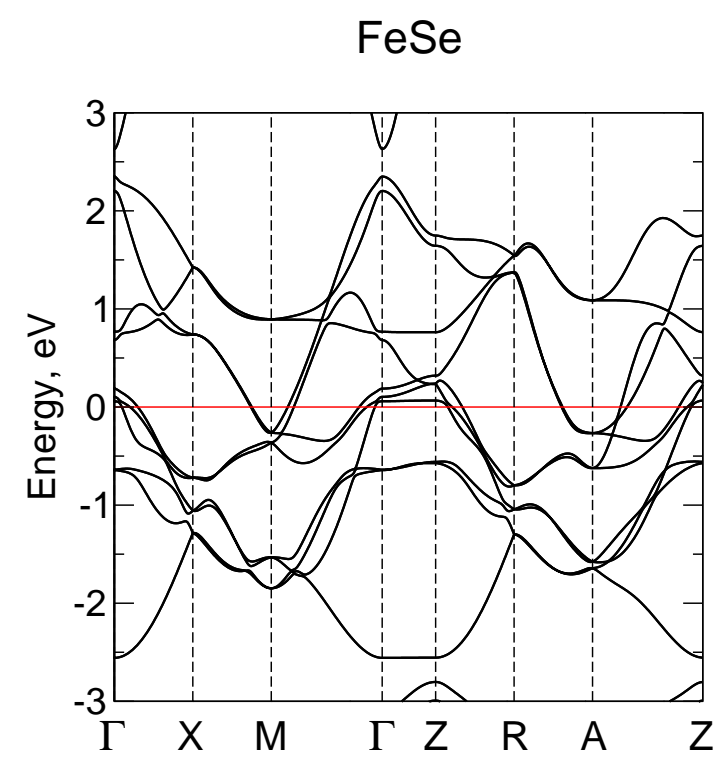

Fig. 4. Band structure of paramagnetic FeSe around $E_{\mathrm{F}}$. The Fermi level position (at $0 \mathrm{eV}$ ) is marked by a horizontal line

and temperature were investigated. The calculations of electronic structures were carried out by a full-potential relativistic linear muffin-tin orbital method (FP-LMTO, code RSPt [16]). The exchange-correlation potential was treated within the local spin density approximation [17] of the DFT. The calculated basic features of the electronic structure for FeSe and FeTe are in a qualitative agreement with the results of earlier calculations [13].

As can be seen in Fig. 4, the present band structure calculations point to the presence of hybridized predominantly $d$-like Fe electronic states close to the Fermi level $E_{\mathrm{F}}$. The chalcogen $p$-states are situated well below $E_{\mathrm{F}}$ and are slightly hybridized with the $\mathrm{Fe} d$-states. As a whole, the electronic structures near $E_{\mathrm{F}}$ are formed within metallic Fe layers, with direct Fe-Fe interactions.

This can be also seen from the calculated densities of states (DOS) $N(E)$ of FeSe and FeTe, which are presented in Figs. 5 and 6 , respectively. In a vicinity of the Fermi level $E_{F}$, the $d$-states of Fe provide the dominant contribution to DOS in the range $-2 \mathrm{eV}$ and $2 \mathrm{eV}$ around $E_{\mathrm{F}}=0$. The $p$ states of chalcogen atoms are predominantly extended in the interstitial region, and their partial contributions to DOS in a vicinity of $E_{\mathrm{F}}$ are substantially smaller for both FeSe and FeTe. As is seen in Fig. 5, the Fermi level in FeSe lies at the steep slope of $N(E)$, at the beginning of a pseudogap of about $\simeq 0.7$ $\mathrm{eV}$. In fact, there is a van Hove singularity in $N(E)$ at about $0.05 \mathrm{eV}$ below $E_{\mathrm{F}}$ (see Fig. 5).

To evaluate the paramagnetic susceptibility of FeSe and FeTe, FP-LMTO calculations of the field-induced 


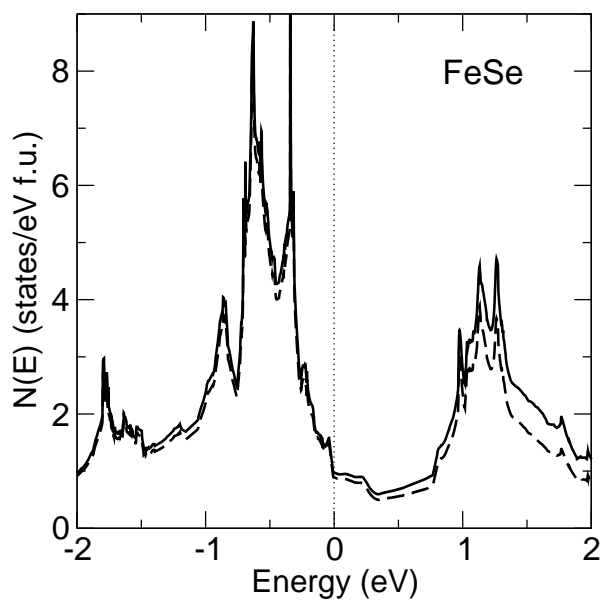

Fig. 5. Total density of states of paramagnetic FeSe around $E_{\mathrm{F}}$ (solid line) and the partial contribution of the iron $d$-states (dashed line). The Fermi level position (at $0 \mathrm{eV}$ ) is marked by a vertical line

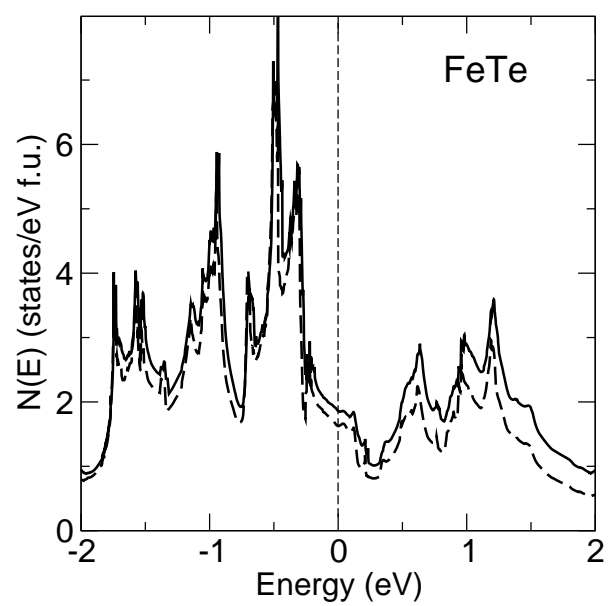

Fig. 6. Total density of states of paramagnetic FeTe around $E_{\mathrm{F}}$ (solid line) and the partial contribution of the iron $d$-states (dashed line). The Fermi level position (at $0 \mathrm{eV}$ ) is marked by a vertical line

spin and orbital (Van Vleck) magnetic moments were carried out within the approach described in [18]. The relativistic effects, including spin-orbit coupling, were incorporated, and the effect of an external magnetic field $\mathbf{H}$ was taken into account self-consistently by means of the Zeeman term:

$\mathcal{H}_{\mathrm{Z}}=\mu_{\mathrm{B}} \mathbf{H}(2 \hat{\mathbf{s}}+\hat{\mathbf{l}})$.

Here, $\mathbf{H}$ is the external magnetic field, $\mu_{\mathrm{B}}$ the Bohr magneton, $\hat{\mathbf{s}}$ and $\hat{\mathbf{l}}$ the spin and orbital angular momentum operators, respectively. The field-induced spin and orbital magnetic moments provide the related contributions to the magnetic susceptibility, $\chi_{\text {spin }}$ and $\chi_{\text {orb }}$.
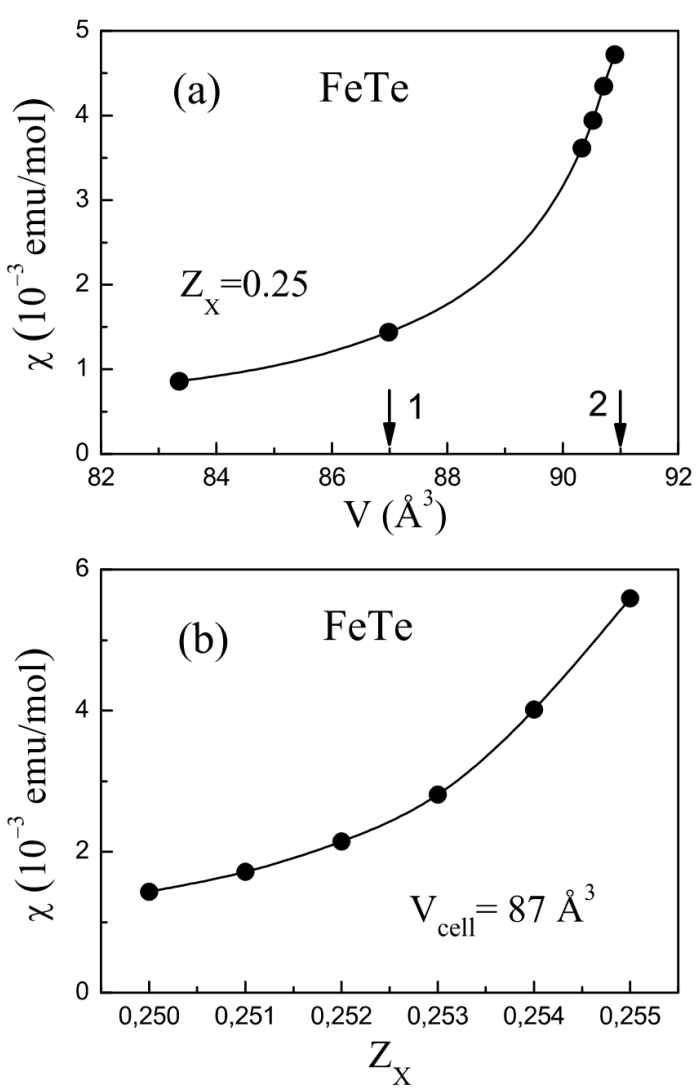

Fig. 7. Calculated paramagnetic susceptibility of FeTe. (a) As a function of the unit cell volume. $Z$ is taken to be 0.25 . The arrows indicate the theoretical (1) and experimental (2) equilibrium volume values. (b) As a function of $Z$ for the theoretical unit cell volume. The $c / a$ ratio is fixed to the experimental ambient pressure value $(1.64,[8])$. Solid lines are guides for the eye

For the tetragonal crystal structure, the components of these contributions, $\chi_{i \|}$ and $\chi_{i \perp}$, are derived from the moments calculated in an external field of $10 \mathrm{~T}$, which was applied in parallel and perpendicularly to the $c$ axis, respectively. The evaluated anisotropy of the orbital contribution $\Delta \chi_{\text {orb }}=\chi_{\text {orb } \|}-\chi_{\text {orb } \perp}$ for FeSe and FeTe amounts to $-0.3 \times 10^{-4}$ and $-0.35 \times 10^{-4} \mathrm{emu} / \mathrm{mol}$, respectively.

It is found that the magnetic response to the external field is very sensitive to the unit cell volume, as well as to the internal parameter $Z$, related to the height of chalcogen species from the Fe plane. The calculated dependences of the susceptibility of FeTe as functions of the volume and the parameter $Z$ are given in Fig. 7. Similar dependences are obtained for FeSe, but its susceptibility is about 10 times lower.

Finally, the thermal effects were taken into account to calculate the temperature dependence of the suscep- 
tibility for FeSe (see Fig. 8). It should be noted that the energy derivative of the Fermi-Dirac distribution function $-\mathrm{d} f / \mathrm{d} E$ comes to the Dirac delta function at low temperatures, having a sharp positive peak at the Fermi energy. This steep behavior of $-\mathrm{d} f / \mathrm{d} E$ has introduced some instability in the numerical calculations of $\chi$, which are seen in Fig. 8.

\section{Discussion}

As seen in Fig. 1, our experimental data points to a gradual increase of the intrinsic magnetic susceptibility in $\mathrm{FeSe}_{1-x} \mathrm{Te}_{x}$ with the Te content by about ten times. For FeSe, a substantial growth of the susceptibility with the temperature was revealed up to $300 \mathrm{~K}$, which indicates the itinerant nature of electronic states of Fe. In fact, this is confirmed by the calculated $\chi(T)$ for FeSe (Fig. 8), which is in a qualitative agreement with the obtained experimental data.

The observed anisotropy of the susceptibility $\Delta \chi$ is large in FeSe and comparable with the averaged susceptibility at low temperatures. If one compares the experimental data in Fig. 2 with the calculated $\chi(T)$ (Fig. 8) and $\Delta \chi_{\text {orb }} \simeq-0.3 \times 10^{-4} \mathrm{emu} / \mathrm{mol}$, then the presence of a substantial anisotropic diamagnetic contribution can be assumed. Actually, the calculated $\chi_{\text {spin }}$ and $\chi_{\text {orb }}$ paramagnetic contributions have to be substantially compensated by a diamagnetic one in order to conform to the experimental data in Fig. 2. Though the sign of the calculated $\Delta \chi_{\text {orb }}$ is consistent with the observed $\Delta \chi$ for FeSe (Fig. 2), only the additional anisotropy could explain the experimental data on $\Delta \chi$. It should be noted that a diamagnetism of conduction electrons $\chi_{\mathrm{L}}$ can be many times larger than the free-electron Landau estimation, and such anomalous anisotropic diamagnetism is determined by the presence of quasidegenerated states with small effective masses at $E_{\mathrm{F}}$ (see, e.g., [19] and references therein).

As is seen in Fig. 7, the tetragonal FeTe compound is on the verge of magnetic instability. Therefore, our field-induced LSDA calculations for FeTe converged to the paramagnetic state only for the reduced values of lattice parameters. Nevertheless, these calculations have allowed us to establish a trend for the effects of the structural parameters $V$ and $Z$ on $\chi$ and to shed light on the nature of the observed pressure effect. Specifically, based on the results of $a b$ initio calculations presented in Fig. 7, it is found that the observed substantial pressure effect on $\chi$ for FeTe can be represented as a sum of two large in size and competing contributions, resulted from the pressure dependence of the structural parameters $V$

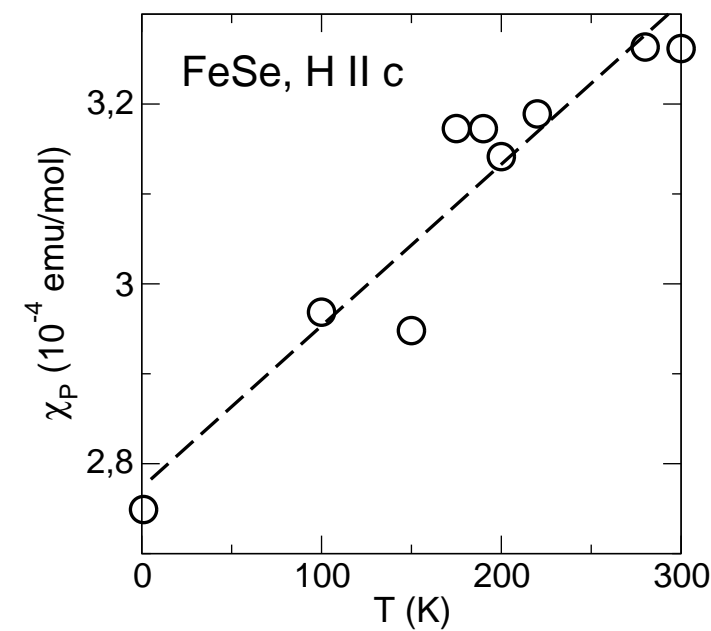

Fig. 8. Calculated temperature dependence of the paramagnetic susceptibility of FeSe. $Z$ is taken to be 0.256 , the unit cell volume and $c / a$ ratio are fixed to their experimental ambient pressure values. The dashed line is a guide for the eye

and $Z$. Using the values of $\mathrm{d} V / \mathrm{d} P(<0)$ and $\mathrm{d} Z / \mathrm{d} P$ $(>0)$ evaluated from $[3,6,12]$, the large experimental value of the pressure effect, $\mathrm{dln} \chi / \mathrm{d} P \simeq 20 \mathrm{Mbar}^{-1}$, can be described.

\section{Conclusions}

The magnetic susceptibility in the series of iron chalcogenides $\mathrm{FeSe}_{1-x} \mathrm{Te}_{x}$ was found to increase gradually with the Te content by about ten times. For FeSe, a substantial growth of the susceptibility with the temperature was revealed, which points to the itinerant nature of the electronic states of Fe. The observed anisotropy of the susceptibility $\Delta \chi$ is large in FeSe and comparable with the averaged susceptibility at low temperatures.

First-principles calculations of the paramagnetic susceptibility of FeSe and FeTe revealed that these systems are in close proximity to the quantum critical point, and this nearness can result in strong spin fluctuations. The calculated paramagnetic susceptibility of FeSe describes qualitatively the experimentally observed rise of $\chi$ with the temperature. The comparison of the experimental and calculated susceptibilities has revealed an additional anisotropic diamagnetic contribution about $-1 \times 10^{-4}$ $\mathrm{emu} / \mathrm{mol}$, which can originate from the diamagnetism of conduction electrons and has its origin in the quasidegenerate electronic states close to $E_{\mathrm{F}}$.

The calculated paramagnetic susceptibility of FeTe revealed a sharp sensitivity to the unit cell volume $V$ and the height of Te species from the Fe plane. It is found that the observed large positive pressure effect on $\chi$ is 
related to a considerable sensitivity of the susceptibility to the structural parameters, particularly to the internal parameter $Z$ determining the dominant positive term. Our results point out that the itinerant magnetism approach is relevant to describe the paramagnetic state of FeSe, but more rigorous calculations of $\chi$ are required for FeTe and the Te rich systems.

This work has been supported by the RussianUkrainian RFBR-NASU project 43-02-10 and 10-0290409, and by NASU Young Scientists Grant 16-2011.

1. F.C. Hsu, J.Y. Luo, K.W. Yeh, T.K. Chen, T.W. Huang, P.M. Wu, Y.C. Lee, Y.L. Huang, Y.Y. Chu, D.C. Yan, and M.K. Wu, Proc. Natl. Acad. Sci. U.S.A. 38, 14262 (2008).

2. E. Pomjakushina, K. Conder, V. Pomjakushin, M. Bendele, and R. Khasanov, Phys. Rev. B 80, 024517 (2009).

3. Y. Mizuguchi and Y. Takano, J. Phys. Soc. Japan 79, 102001 (2010).

4. A.V. Fedorchenko, G.E. Grechnev, V.A. Desnenko, A.S. Panfilov, S.L. Gnatchenko, V.V. Tsurkan, J. Deisenhofer, H.-A. Krug von Nidda, A. Loidl, D.A. Chareev, O.S. Volkova, and A.N. Vasiliev, Low Temp. Phys. 37, 83 (2011).

5. D. Braithwaite, B. Salce, G. Lapertot, F. Bourdarot, C. Marin, D. Aoki, and M. Hanfland, J. Phys.: Condens. Matter 21, 232202 (2009).

6. S. Margadonna, Y. Takabayashi, Y. Ohishi, Y. Mizuguchi, Y. Takano, T. Kagayama, T. Nakagawa, M. Takata, and K. Prassides, Phys. Rev. B 80, 064506 (2009).

7. G. Tsoi, A.K. Stemshorn, Y.K. Vohra, P.M. Wu, F.C. Hsu, Y.L. Huang, M.K. Wu, K.W. Yeh, and S.T. Weir, J. Phys.: Condens. Matter 21, 232201 (2009).

8. S. Li, C. de la Cruz, Q. Huang, Y. Chen, J.W. Lynn, J. Hu, Y.-L. Huang, F.-C. Hsu, K.-W. Yeh, M.-K. Wu, and P. Dai, Phys. Rev. B 79, 054503 (2009).

9. B.C. Sales, A.S. Sefat, M.A. McGuire, R.Y. Jin, Y.D. Mandrus, and Y. Mozharivskyj, Phys. Rev. B 79, 094521 (2009).

10. J. Yang, M. Matsui, M. Kawa, H. Ohta, C. Michioka, C. Dong, H. Wang, H. Yuan, M. Fang, and K. Yoshimura, J. Phys. Soc. Japan 79, 074704 (2010).

11. A.S. Panfilov, Physics and Technics of High Pressures (in Russian) 2, 61 (1992).
12. J.N. Millican, D. Phelan, E.L. Thomas, J.B. Leao, and E. Carpenter, Solid State Commun. 149, 707 (2009).

13. A. Subedi, L. Zhang, D.J. Singh, and M.-H. Du, Phys. Rev. B 78, 134514 (2008).

14. M.-J. Han and S.Y. Savrasov, Phys. Rev. Lett. 103, 067001 (2009).

15. A.N. Yaresko, G.-Q. Liu, V.N. Antonov, and O.K. Andersen, Phys. Rev. B 79, 144421 (2009).

16. J.M. Wills, M. Alouani, P. Andersson, A. Delin, O. Eriksson, A. Grechnev, Full-Potential Electronic Structure Method (Springer, Berlin, 2010).

17. U. von Barth and L. Hedin, J. Phys. C: Solid State Phys. 5, 1629 (1972).

18. G.E. Grechnev, R. Ahuja, and O. Eriksson, Phys. Rev. B 68, 64414 (2003).

19. G.E. Grechnev, A.V. Fedorchenko, A.V. Logosha, A.S. Panfilov, I.V. Svechkarev, V.B. Filippov, A.B. Lyashchenko, and A.V. Evdokimova, J. Alloys Compound. 481, 75 (2009).

Received 20.09.11

\section{ЕЛЕКТРОННА СТРУКТУРА І МАГНЕТИЗМ НАДПРОВІДНИКІВ, ЩО МІСТЯТЬ Fе}

Г.Є. Гречнєв, А.С. Панфілов, О.В. Федорченко, В.О. Десненко, І.П. Журавлъова, С.Л. Гнатченко, Д.А. Чареєв, О.С. Волкова, О.М. Васильєв

$\mathrm{P}$ е $з$ ю м е

Для новітніх надпровідників системи $\mathrm{FeSe}_{1-x} \mathrm{Te}_{x}$ виконано розрахунки електронних структур з перших принципів з метою пояснення експериментально знайдених аномальних магнітних властивостей у нормальному стані. Розрахунки показали, що сполуки $\mathrm{FeSe}_{1-x} \mathrm{Te}_{x}$ близькі до магнітної нестабільності з домінуючим посиленим спіновим парамагнетизмом. Встановлено, що магнітна сприйнятливість монотонно зростає зі збільшенням концентрації Те. Температурні залежності магнітної сприйнятливості $\chi$ i ïi анізотропія $\Delta \chi$ досліджено для $\mathrm{FeSe}, \mathrm{i}$ встановлено зростання сприйнятливості з температурою в діапазоні 4,2-300 К. Для FеТе виявлено істотне збільшення $\chi$ під тиском. Розрахунки парамагнітної сприйнятливості виявили їі сильну залежність від об'єму елементарної комірки $V$ i, особливо, від відстані між шарами заліза та халькогена. Проведені розрахунки пояснюють експериментальні залежності $\chi(T)$ і $\chi(P)$ для FeSe i FeTe відповідно. 